painful nicturition, but had little influence on the pyelitic symptoms. The extreme exhaustion of the patient seemed to me to be a contraindication to immediate nephrotomy, wherefore I tried to keep the patient up with stimulation, at the same time giving hexamethylenamine internally. Three weeks afterward, when the vesical wound was thoroughly healed, I resorted to nephrotomy. The large triangular calculus was tightly imbedded in the parenchyma, so that it had to be mostly shelled out with the knife. 'The patient was discharged from the hospital four weeks afterward.

A small calculus was found near the lower surface of the large renal concretion. It would most probably have formed the nucleus for a third calculous formation in the bladder if it had been removed. The calculi consisted of oxalate of lime and the outer layer showed a smaller degree of density than the inner.

In another case of great skiagraphic interest, the patient was operated on at the Postgraduate Hospital. The patient, a woman of 36 , began to suffer from violent pain in the right lumbar region thirteen years ago. Examination of the urine revealed the presence of pus. Nephrotomy disclosed the presence of a calculus of moderate size surrounded by pus. It was supposed, then, that the calculus was the source of the suppuration, and consequently a speedy recovery was expected. This expectation was not realized, a fistula remaining. The patient improved considerably, but the fistula did not close, so that after a year had elapsed, nephrectomy was advised, to which the patient, however, did not submit because her general condition had become very good in the meanwhile and the discharge had decreased.

About a year later the fistulous tract closed, more pus appeared in the urine and the left side became painful. Left-sided nephrotomy was done, a large abscess being evacuated. The patient again improved and the upening closed, but the pyuria continued, the patient's condition varying considerably.

When I saw her for the first time I suggested skiagraphic examination beside the other modern diagnostic means (cryoscopy, ureteroscopy), but the patient said: "There is no necessity for examining for nephrolithiasis, because the stone I had was removed by the surgeon." I mention this as an interesting and misleading argument. I, of course, insisted on my proposition, and the skiagraph showed the faint outlines of four calculi in the right lumbar region. A second exposure with the diaphragm showed them very distinctly.

The calculi had to be shelled out with difficulty, the irregular surface forming an impediment. The perirenal structures were matted and hardened, a natural consequence of the long-standing suppuration and inflammation. The adhesions were so dense that the kidney could not be lifted out, and great care had to be exercised during the whole procedure. This observation is another point against the formation of normal blood circulation after decapsulation. One stone had a sharp triangular edge. Recovery took place in two weeks, the patient being able to go around at that time. Three weeks after the operation febrile elevations began and the left side became painful. An abscess formed which was promptly discharged. Exploration of the abscess cavity revealed the presence of a long sinus leading to the opposite side just in front of the spinal column. The patient improved slowly and is up at the present writing, the thorough closing of the wound being cxpected in a short time.

The epicrisis of this case suggests that nephrolithiasis existed at the time of the first operation, when the
Roentgen method was unfortunately not yet available. The very able surgeon who operated at that time undoubtedly examined the kidney carefully, but was unable to palpate the calculi through the parenchyma. The advice given later, when the fistula did not close, suggests that he thought the kidney to be in a state of destruction rather than assumed the presence of the stone, which under the cireumstances would have been most natural. Under the most careful wound treatment and the good care which the intelligent patient exercised, the fistula closed, but the pus became retained in the depth and probably found a way to the opposite side which necessitated the incision on the left side. It is evident that if the Roentgen method had been available at the time of the first operation, the remaining calculi which penetrated the whole kidney would have been recognized and removed at once, thus sparing years of suffering. It did not only reveal their number, but also their size and shape, and-last but not least-localized them. After the surgeon has removed the calculi, which are easily identified with those of the plate, no further searching is required, as it was imperative in former years, when the kidney had to be nearly dissected in order to ascertain whether there were any additional calculi left.

\section{INFLAMMATION OF THE EYE DUE TO THE TOXINS OF THE GONOCOCCUS.}

SWAN M. BURNETT, M.D., Pir.D.

Professor of Ophthalmology and Otology, Medical School, Grorgetown University.

WASHINGTON, D. C.

IRITIS.

Under the name of metastatic ophthalmia, or some other such synonym, inflammation of the iris has long been recognized among ophthalmologists as a possible accompaniment of gonorrheal urethral infection. There is scarcely a treatise on diseases of the eye since $\mathrm{Mc}$ Kenzie's time, but speaks of it in a more or less extcoced manner.

The iritis is commonly regarded by ophthalmic authorities, as a part of an intoxication which manifests itself uncler the form of rheumatism; at least, few cases have been reported which have not been associated with greater or less pronounced symptoms of a rheumatic nature-usually affecting the joints.

Whether the intoxication in these instances is from an entrance into the circulation of the gonococcus itself or from the product known as the toxins, has not been definitely settled to the satisfaction of all. Further investigation and observation along this line are needed. The fact of the association of the diseases with gonorrheal infection, however, is no longer disputed.

As to the frequency of gonorrheal iritjs, all authorities are not agreed. By some it is considered as extremely rare. De Lapersonne ${ }^{1}$ of Paris, for instance, who is one of the latest writing on the subject, states that he sees only one or two cases in every 7,000 new patients at the Hotel Dieu. Some others, however, seem to regard it as rather common. This discrepancy may be accounted for by the fact that the complication is more frequent in some localities than in others, or, what is more probable, by a failure on the part of some clinjcians to inquire into the existence of gonorrhea in the cases of iritis which come under observation. It

1. Archiv. D'ophth., March, 1905 
is a fact not without bearing on the point of frequency, that from the large clinic for genitourinary disease at the Central Dispensary and Emergency Hospital, where my own clinic is located, there has not been a single case of gonorrheal iritis referred to my service during the last twenty-five years, while cases of iritis from syphilis have come to me from that clinic in large numbers. It may be of interest to know that the genitourinary clinic is largely recruited from the negroes, among whom gonorrhen is most rife.

The forms under which gonorrheal iritis may appear are various. It may be the so-called spongy iritis with a deep anterior chamber and dilated pupil, or any of the plastic varieties with various kinds of exudate, and even the hemorrhagic form has been mentioned. The socalled spongy iritis, such as was present in the case to be described, while one of the rarest forms of iritis to be met, generally finds its place here, I think, with greater comparative frequency than the other forms. As another distinctive feature of gonorrheal iritis may be mentioned its tendency to recurrence during the same or subsequent attacks of gonorrhea. The same holds true of the common rheumatic form. The disease may be very intense in its manifestation or mild and slow in its progress. Almost without exception, both rheumatism and iritis show themselves during the declining stage of the urethral disease, quite contrary to what would be expected. One or both eyes may be affected at the same time or in succession.

It seems singular and altogether quite unaccountable that while gonorrheal iritis finds at least a mention in practically every text-book of importance on diseases of the eye, it seems to be completely ignored in all the treatises on genitourinary diseases which I have been able to consult, with the exception of two American textbooks. ${ }^{1}$

It is, therefore, not without justification that the subject is brought more closely to the attention of the general practitioner and genitourinary surgeons among whose patients the disease shows itself primarily, and in whom a correct diagnosis should first be made, and, of even more importance, a proper treatment promptly begun. A careless or not sufficiently informed practiiioner, not being aware of the possibility of iritis as a complication of gonorrhea would naturally look on any redness or infection of an eye as a beginning coniuuctivitis due to an external infection, and treat it as such with astringents and antiseptics, probably for some days, so that by the time the real condition becomes known irreparable damage would already have been

1. An examination was made of all the treatises on genito pinary diseases to be found in the library of the Surgeon-General's urinary diseases to be found in the
cfice with the following result:

Morrow : "System of Disnases of the Genitourinary Organs," New York, 1894.. No mention. Stokes, F. W.: "Diseases of the Genitourinary Organs," London, 1892. No mention. Taylor, $R$ W.: "A 'Treatise on Genitourinary and Venereal Diseases," New loik and philadelphia, 10n4. No mention. Fuller, Eugene "Diseases of the Genitourinary System," New York, 1892. Simply "Diseases of the Genitourinary system," Nenitourinary Diseases, Lon inentions it. Wo mention. Acton, w. "The Diseases of the Genito ton, 18.4. No mention. Acton, W.: "The Diseases of the Gentto "Iniseases of the Gentitourinary Organs," New York, 1884. No "Iiseases of the Gentourinary Organs," New York, 1884 . No mention. "American Text-Book of Genitourinary Organs." Philadelphia. No mention. Black, D. C.: "Diseases of the Genito-
lirinary Organs," Philadelphia, 1872. No mention. Guthrie, G. J.: "Grinary Organs," Philadelphia, 1872. No mention. Guthrie, G. J. : II.: "Diseases of the Genitnurinary Organs," London, 1895. No inention. Hyde and Montgomery: "Sypbilis and Venereal Dis eases," Ihiladelphia, 1900. Quite a full ascount of eye affec tions occurring in gonorrhea under the heading of gonorrheal rheumatism-not, however, placed in the index separately.

E. L. Keyes and E. L. Keves, Jr. "Surgical Diseases of the Genito-urinary Organs," New York, 1903. A full account, similar to the above, of iritis and conjunctival affections in gonorrhea, considered under the heading of gonorrheal rheumatism. Not indexed separately. done. As is well known, it is more than usually necessar? that in iritis the use of a mydriatic be begun early in "orier to secure at once as full a dilatation of the pripil as possible-since, otherwise, permanent adhesion to the anterior capsule of the lens or complete closure of the pupil is almost certain to occur. A day or two, or even a few hour's' delay, may mark the line between the preservation of good vision and partial or even complete blindness. As a clinical contribution to the stud?y of this form of disease the following history is offereri :

Patient.-J. A. D., a young white man aged 22 , came to me on Oct. 6, 1904, complaining of his eyes, which he said felt uneomfortable but not painful, and as he had been suffering from gonorrhea for some three weeks, he was fearful that this might be the beginning of a gonorrheal conjunctivitis, as he was aware that "gonorrhea often affected the eyes."

Examination and Treatment.-As the conjunctiva was clear and the pupils responded promptly to light, I assured him that there was no inflammatory trouble, but, as an examination revealed myopic astigmia of $0.5 \mathrm{D}$. with the rule, he was or dered correcting glasses, which gave $V=5 / 5$ in both eyes.

Subsequent History.-On October 7 there was some slight injection of the right eyeball, the vessels being fine and coming up to the corneal edge; no mucus or pus. There was no pain and the pupil reacted well to light. He was ordered to make hot applications to this eye and to abstain from work. This condition continued about the same with clear media and a normal fundus until October 12, when the bulbar congestion increased, but with no complaint of pain. Atropin gr. ii to aqua $3 i$, was then ordered in addition to the hot applications

October 13: When he awoke he found that his vision was almost entirely gone. An examination revealed great muddiness of the anterior chamber and a rigid but fair-sized pupil. The fundus could not be made out in any detail, only a dull diffuse redness being visible under the illumination. An examination with the binocular magnifier showed many fine fibers coming out from the pupillary space and reaching to the posterior surface of the cornea. The strength of the atropin was increased to gr. iv to aqua $3 i$, a drop in the eyelid three times a day, and dionin (ethyl-morphin hydrochlorate), 5 per cent., was used. The diagnosis of spongy iritis was made.

October 14: The mass in the anterior chamber had assumed its characteristic gelatinous appearance, filling the anterior chamber except for a small section above. The edge of the exudate, as is always tne case in spongy iritis, was sharply marked and the mass could be seen to extend from the iris to the cornea, occupying the entire depth of the chamber. The size of the pupil remained about the same.

October 15: There was a still further diminution in the exudate, principally above, so that the pupil was somewhat uncovered.

October 16: All that remained of the exudate was a band about $2 \mathrm{~mm}$. broad with sharp edges, occupying the center of the pupil from above downward. It had some fine offshoots connecting it with the pupillary edge above and below. The iris itself had a clean, unswollen look, the natural rugæ showing up plainly. The pupil was larger and vision was returning. There was still no acute pain complained of, only some slight frontal headache. The conjunctival injection, while pronounced, was not excessive and there was little or no chemosis.

October 17: The pupil was dilated ad maximum and the exudate was found to be all gone except a few spots on the anterior capsule of the lens. The fundus could be seen but rather dimly.

October 23: The lens was entirely clear and the fundus only slightly hazy, $\mathrm{V}=5 / 20$. In the course of ten days more every vestige of the disease had disappeared and vision was normal. Since then there has bcen no further trouble in either eye. The patient's gonorrhea has also disappeared.

Special inquiry was made as to the manifestations of rheumatism and it was found that some days before the appearance of the uncomfortable feeling in his eye 
he hat a pain and some swelling in the joints of the big toe of the left foot. While not severe, these symptoms ware present during the whole course of the eye affection.

\section{CONJUNCTIVITIS.}

The existence of conjunctivitis due to an endogenous infection of gonorrheal toxins is certainly rare, and is questioned in many quarters. Before the era of close bacteriologic study it is quite possible that cases of this kind may have escaped observation, since it would be only natural to refer any conjunctival inflammation with a mucopurulent or purulent discharge to an external infection. The case here reported might easily have been classed as an ordinary gonorrheal infection of the conjunctiva had I not followed my invariable custom of examining all conjunctival discharges as to their bacteriologic character.

Patient.-R. C., white, aged 25, came to me on. May 22 , 1905 , with a double mucopurulent conjunctivitis.

Examination.- There was but slight swelling of the lids. The cornea was clear and the iris and other internal structures normal. He first noticed a discharge from his eyes two days ago. As he confessed to having a slight gonorrhea (a second attack), an external infection was considered almost certain. Nevertheless a smear of the discharge was taken. He was given a cleansing wash, to be used three times a day. An examination of the slide by Dr. Le Merle at the Lionel laboratory at the Emergency Hospital failed to reveal any organisms of any kind. The specimen was pure mucopus.

Course of the Disease.-May 24: There was quite considerable thickening of the conjunctiva of the lids. The eyeball itself was not entirely free from infection, and there were a few fine ecchymoses of the conjunctiva near the cornea in both eyes. The discharge was about the same-not creamy, but stringy and in rolls. On May 28 another careful examination of the discharce was made, with the same result as before-no organisms of any kind whatever were found. By May 30 the discharge was much less, and the infection of the ball quite gone.

Remarks.-By June 1 the eyes were practically free from discharge, though there yet remained some thickening of the conjunctiva of the lids, particularly the lower retrotarsal folds. An inquiry into his history revealed the fact that when he had his first attack of gonorrhea, two years ago, about three weeks after the beginning and when it was in the stage of decline. he was seized with rheumatism in the left foot which laid him up for three weeks. During his present attack he has had pain and tenderness of the patellar ligaments of the left knee-not severe, but sufficient to keep him reminded that he has a knee joint.

I am aware, of course, that it may be charged that there is little evidence, beyond that of coincidence, that the conjunctival inflammation was due to an endogenous affection. An external affection, however, I think, can be positively excluded, and we should at least learn from this the importance of a bacteriologic examination in every case of purulent conjunctivitis occurring in gonorrheic patients. If no gonococci are found, the prognosis is quite different from that when their presence is demonstrated.

External inflammations of the eye in gonorrheic infection hare been observed and commented on by both ophthalmic and genitourinary authors. They have generally heen described as of a mild type.

Taylor, who is one of the few who mention it at all, in his "Text-Book on Genitourinary Diseases," 1904, has characterized it as "serovascular conjunctivitis." Others evidently regard it as a form of scleroconjunctival affection. No author I have been able to consult has mentioned the pronounced mucopurulent form here describel. While it is quite true that most of the complications of general gonorrheal infection show themselves under the form of the so-called rheumatic inflammation, that is a plastic form affecting the fibrous structures, there are certain instances in which it assumes the purulent form, as in cases of endocarditis that have been reported. A more careful examination and observation of cases of purulent conjunctivitis in gonorrheic subjects would throw much needed light on this subject, which has an important pathologic, as well as a highly practical interest.

\section{THE MEDICAL FEATURES OF THE PAPYRUS EBRRS.* \\ CARL H. VON KLEIN, A.M., M.D. CHICAgo.}

This is an age of inquiry in researches and excavation, with a constant craving for some new development to assist us in our studies.

The last hundred years is the Niobe of civilization, not only in inventions and productions, but in the remarkable revelations which have materially assisted us in the wonderful development in science. However, whatever we have done to advance the history of civilization was not done by an easy task.

When we look through the magnificent works of discoverers and pioneers, the fruit of hardship, trial and labor, we can not fail to be infected with some of the enthusiasm which animated those who were endowed with superior intelligence and the gift of knowledge to bring forth hidden treasures from the inmost bosom of the earth, and to transmit them to future generations. Of one of these it may well be said: "From the Orient to the Occident," great be thy name (George Ebers).

The Anglo-Saxon nations, though renowned for deep thinking, and philosophizing in every branch of science and art, can not boast of a scholarship in bringing forth the first literature of the science of medicine. A third of a century has elapsed since George Ebers revealed the pages on medicine which were written some seven thousand years ago, and which were concealed for nearly four thousand years between the legs of a mummy. With the assistance of the learned. Ludwig Stern and other Egyptologists, Ebers published the fact that Hippocrates of Cos, who for twenty-three hundred years has been known to the world as the "Father of Medicine," and as an original observer, no longer possesses this distinction. It has been wrested from the ancient Greek by the discovery of this papyrus of a date so remote as almost to place Hippocrates within the ranks of modern physicians.

However great the appreciation we may manifest, it is but strictest justice to do homage to the zeal, the remarkable ability and indefatigable activity of this German scholar, whose name is attached to that elaborate work, the "Papyrus Ebers."

The revelation of this important document merits assuredly all praise. In the Ebers Papyrus we have a monument of ancient culture before us, whose medical and historical value is inestimable, as may be seen from the description of its contents.

In this complication we have the most important medical treasure of the Egyptians before us, in a more complete form than any other known work. We learn much, too, of the anatomic, physiologic, pathologic and pathologico-anatomic conception of that time. Further-

* Delivered before the Thirtieth Annual Session of the American Academy of Medicine at Chicago, 1905. 